\title{
Analytic Simulations of Packaging Cardboards with Non-Rigid Adhesives
}

\author{
R. Hussein, Ph.D., P.E. \\ State University of New York, NY, USA \\ email:ezpsc@yahoo.com
}

\begin{abstract}
The understanding of the cardboard performance is necessary to the design of packaging containers and the protection of their contents for safe deliveries. The use of adhesives is unavoidable in the manufacturing of the cardboards. Like all materials, the adhesives have finite stiffness but when used in the literature, they are assumed perfectly rigid. This study changes this assumption by using the real properties of adhesives. A closed-form solution for cardboard panelsassembled withnon-rigid adhesives, and subjected to edgewise loading is presented. The solution satisfies the equilibrium equations of the layers, the compatibility equations of stresses and strains at the interfaces, and the boundary conditions. To investigate the effects of the finite values of adhesivestiffness on the responses, numerical evaluations are conducted. The results obtained have shown that the adhesive stiffness has a strong effect on the performance. Beyond a certain level of stiffness, the usual assumption of perfect bonding used in classical theories is acceptable. This could provide an answer to what constitutes perfect bonding in terms of the ratio of the fluted layer, or simply flute, stiffness to the bonding stiffness.
\end{abstract}

Key Words: Adhesives, Analysis, Bond, Cardboards, Compression, Corrugated, Delamination, Edgewise load, Flute, Interlayer, Liner, Packaging, Panels, Plates, Shear, Stiffness, Strength.

$* * * * *$

\section{General}

\section{INTRODUCTION}

The revenues of the global packaging industry are projected to increase from $\$ 851$ billion in 2017 to $\$ 980$ billion in 5 years. In this growing industry, each manufacturer faces its individual unique challenges but shares a common goal with all others; i.e. to deliver the packages safely to their destinations. This can't be achieved without the necessary knowledge to understand the performance under the primary compressive load during shipping and storage.In general, corrugated cardboardpanels, referred here as panels, comprise a liner(s) and a flute that bonded together using adhesives. The panel styles include single liner, single wall, double wall, and triple wall. The purpose of the liner(s) (also known in the literature as face and skin) is to carry normal stresses resulting from in-plane deformation whereas the flute (also known in the literature as core) carries shear stresses, keeps the liners apart, and is responsible for the integrity of the board to protect the contents of the corrugated containers.

Nowadays, significant advances in the development of biorenewable and biodegradable materials have placed them at the forefront of research and development for alternatives to the environmentally unfriendly materials $(3,7,11,12,22,24,28,29,30$, and 32$)$. They have been provenviable for high-performance in harsh loading conditions(2, 23, 31, 33), the use of natural and synthetic fibers(24, 28, and 29), and the applications of natural adhesives (3 and 8).

Although corrugated board has been used for over a century, research on the panels has been lagging behind its industrial applications and the literature lacks adequate studies on the effects of adhesives on the performance of panels. For example, failures have been reported in the interfaces between the liners and flutes where the adhesive is applied, as shown in Fig. 1. To narrow the gap of the missing information, the author has completed some analytic and experimental studies (19 and 20) in this area although more work is needed. This paper thus revolves around the existing gape between the fundamental science and the industrial applied technologies. It presents an accurate analytic model and solution to investigate and characterize the effects of adhesives on thepanels. This advances the state of knowledge, enhances the clear understanding of how these panels perform and the key governing variables, and foster future frameworks in the field. 

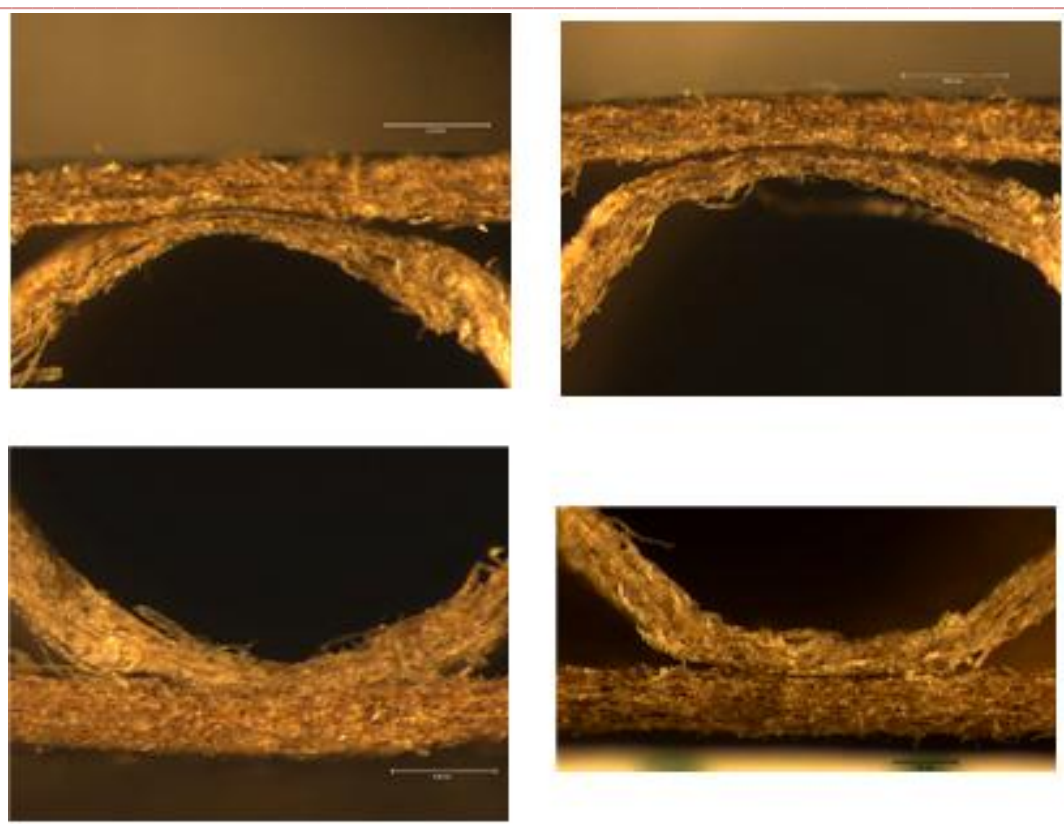

Fig. 1 Failures of Bonding in Corrugated Cardboard Containers

\section{Analytic solutions}

Existing analytic and experimental methods have invariably assumed perfect bonding between layers (1, 25, 26, 23, 31, and 33). Nevertheless, interlayer slips do occur because of the finite bonding stiffness; the bonds creepunder sustained loads, and environmental effects. The high local interlayer shear stress due to applied loads may contribute to an answer of the many delamination problems in structural panels.

Analysis of wood joist floor systems, taking into account interlayer shear deformations due to adhesives, was done by Goodman et al. $(4,5,6$, and 32). In that study, the wood layer were assembled with nails or by gluing their ends, and although the interlayer slip in this system was accounted for in the analytical model, transverse shear deformations were neglected. The interlaminar shear due to plane stress was investigated analytically by Puppo and Evensen (27); and with the finite element method by Isakson and Levy (21).

Very few publications deal with the responses of panels with interlayer slip or orthotropic materials. In a series of analytic and experimental studies, the author has investigated the performance of suchpanels with interlayer slips and under various loads (14, $15,16,18,19$ and 20). In those investigations, many common assumptions from the literature have been replaced with realistic ones such as the use of adhesives having finite stiffness; the effects of flute properties and shear deformations on the panel deformations and stresses; the permission of liners to deform in their own planes. There remains the problem of orthotropic panels, due to corrugation, with interlayer slip and under edgewise loads.

This paper presents an analytical solution of panels with interlayer slips and under edgewise loads. The solution satisfies the equilibrium equations of each layer and the compatibility of deformations at the interfaces. The objective is to ascertain the effect of interlayer slips on the performance of panels due to edgewise loads which are the primary loads for corrugated cardboards during storage, handling, and transportation.

\section{DESCRIPTION OF PROBLEM}

Many studies in this area transformed theflute to an equivalent homogenous one with effective material properties. Consider a panel of span $2 \mathrm{a}$ and width $2 \mathrm{~b}$, subjected to in-plane biaxial loads as shown in Figure2. The liners are of equal thickness $\mathrm{t}_{\mathrm{f}}$. The flute of a thickness $2 \mathrm{t}_{\mathrm{c}}$. The modului of elasticity of the flute and liners are $\mathrm{E}_{\mathrm{cx}}, \mathrm{E}_{\mathrm{cy}}$ and $\mathrm{E}_{\mathrm{fx}}$ and $\mathrm{E}_{\mathrm{fy}}$, respectively.The shear modului of the flute are $G_{c x y}, G_{c x z}$ and $G_{c y z}$. The adhesive between the liners and flute has finite stiffness $K_{x}$ and $K_{y}$. The load applied to the panel shown in Figure 2 can be on the liner(s), flute, or on both components. 


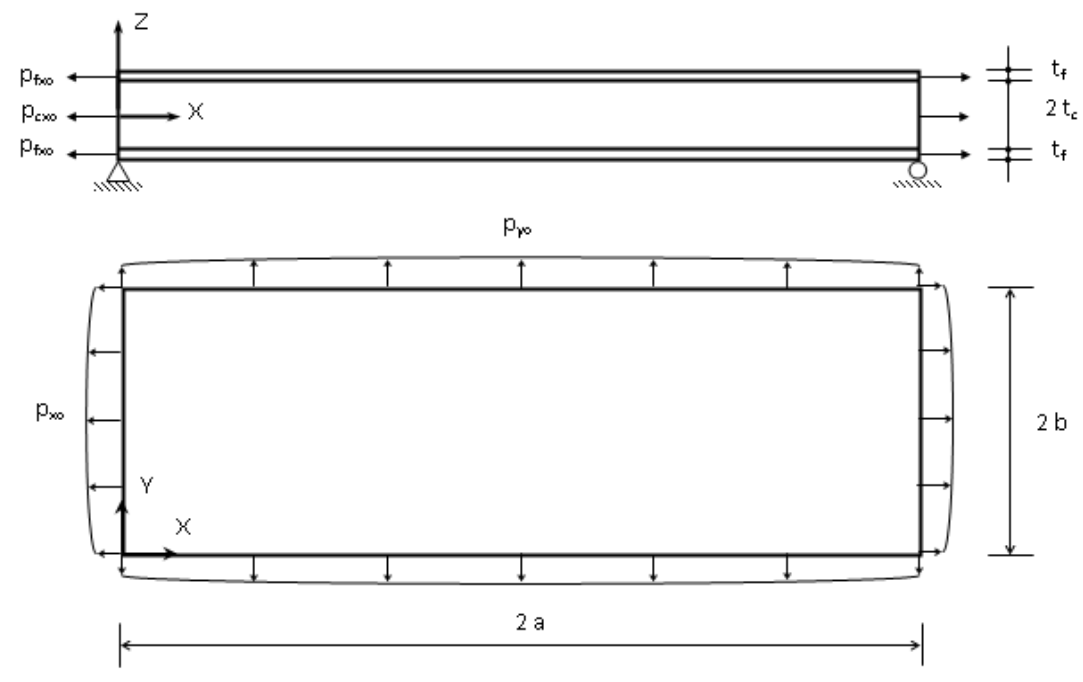

Fig. 2 A Typical Plate

\section{ANALYTICAL DEVELOPMENT}

\section{General}

This kind of problem has been attacked using the fundamentals of theory of elasticity $(9,10,13,14,15,16,17,18)$. Generally, equations are set up to define the equilibrium of the separate liners and of the flute and to prescribe the necessary continuity between the liners and the flute. The result is a set of differential equations which may be solved in particular cases for the quantities of interest. In that kind of problem, the analytic investigation is sufficiently complex and due to the existence of non-zero shear strains in the flute and adhesive, and of the direct strains in the flute.

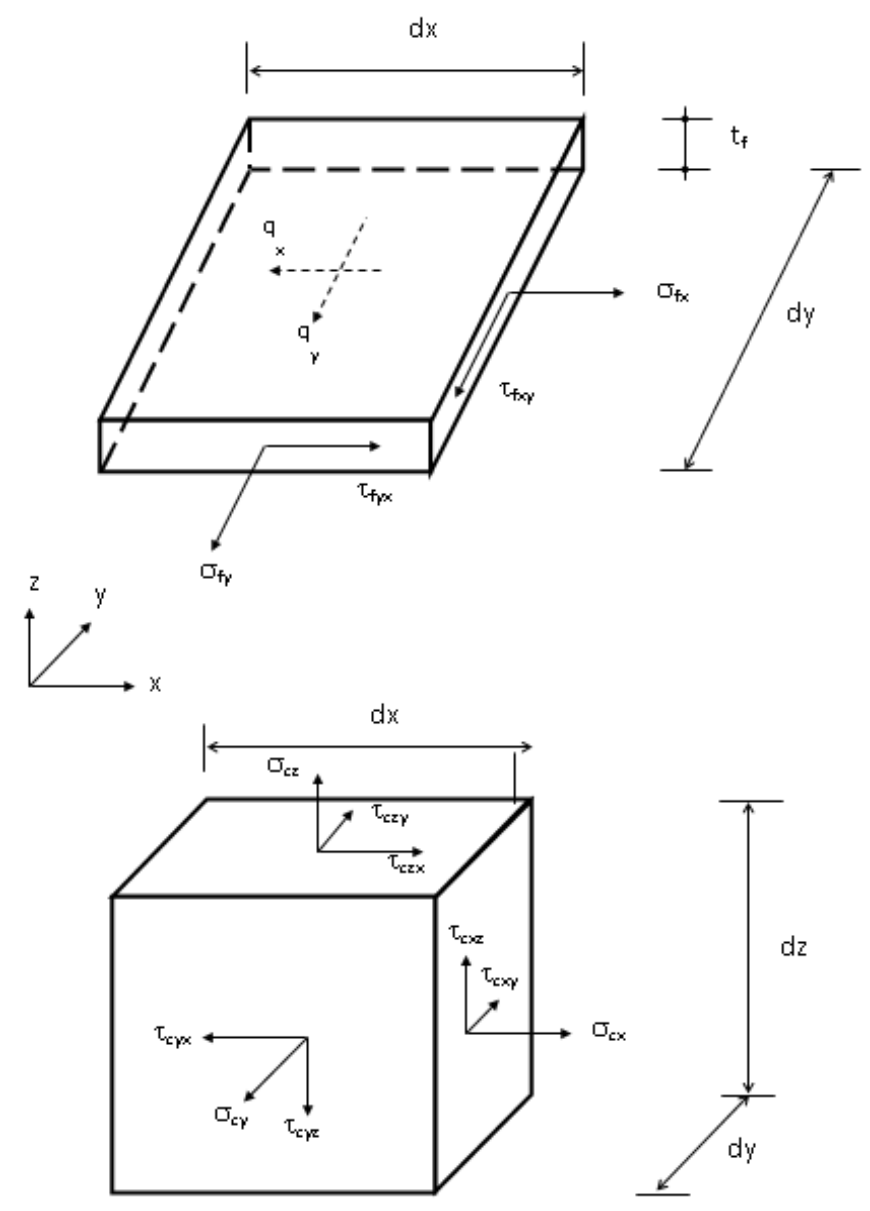

Fig. 3 Stress State in Plate's Element 
The stress state in the liners and fluteelements is shown in Figure 3 . The equilibrium of the liner element requires that

$\frac{\partial \sigma_{f x}}{\partial x}+\frac{\partial \tau_{f y x}}{\partial y}-\frac{q_{x}}{t_{f}}=0$

$\frac{\partial \sigma_{f y}}{\partial y}+\frac{\partial \tau_{f x y}}{\partial x}-\frac{q_{y}}{t_{f}}=0$

in which

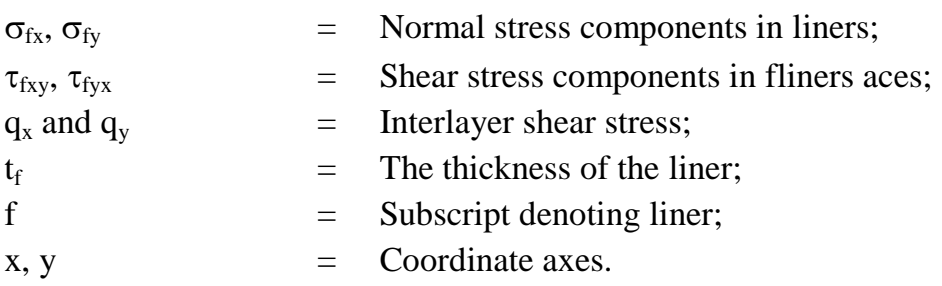

The state of stress in the flutemust satisfy the following equilibrium equations.

$$
\begin{aligned}
& \frac{\partial \sigma_{\mathrm{cx}}}{\partial \mathrm{x}}+\frac{\partial \tau_{\mathrm{cyx}}}{\partial \mathrm{y}}+\frac{\partial \tau_{\mathrm{czx}}}{\partial \mathrm{z}}=0 \\
& \frac{\partial \sigma_{\mathrm{cy}}}{\partial \mathrm{y}}+\frac{\partial \tau_{\mathrm{cxy}}}{\partial \mathrm{x}}+\frac{\partial \tau_{\mathrm{czy}}}{\partial \mathrm{z}}=0
\end{aligned}
$$

in which

$$
\begin{array}{ll}
\sigma_{\mathrm{cx}}, \sigma_{\mathrm{cy}}, \sigma_{\mathrm{cz}} & =\text { Normal stress in the flute; } \\
\tau_{\mathrm{cxy}}, \tau_{\mathrm{cyz}}, \tau_{\mathrm{czx}} & =\text { Shear stress in the flute; } \\
\mathrm{c} & =\text { Subscript denoting flute. }
\end{array}
$$

The normal stress components in the liners and flutemust also satisfy the overall equilibrium equations, which are

$$
\begin{aligned}
& 2 \operatorname{tf} \int_{y=0}^{y=2 b} \sigma_{f x} d y+\int_{y=0}^{y=2 b} \int_{z=-t c}^{z=t c} \sigma_{c x} d y d z+\int_{y=0}^{y=2 b} p_{x} d y=0 \\
& 2 \operatorname{tr} \int_{y=0}^{y=2 a} \sigma_{\text {fy }} d x+\int_{y=0}^{y=2 a} \int_{z=-t c}^{z=t c} \sigma_{c y} d x d z+\int_{y=0}^{y=2 a} p_{y} d x=0
\end{aligned}
$$

wherep $\mathrm{x}_{\mathrm{x}}$ and $\mathrm{p}_{\mathrm{y}}$ are the applied edge loads.

At the interfaces between the fluteand the skins, the stresses and strains must be compatible. The compatibility equations in terms of stresses are

$\mathrm{q}_{\mathrm{x}}=\left.\tau_{\mathrm{czx}}\right|_{\mathrm{Z}= \pm \mathrm{t}_{\mathrm{c}}}$ 
$\mathrm{q}_{\mathrm{y}}=\left.\tau_{\mathrm{czy}}\right|_{\mathrm{z}= \pm \mathrm{t}_{\mathrm{c}}}$

In terms of strains, the compatibility equations are written as

$\frac{\partial \Delta_{\mathrm{x}}}{\partial \mathrm{x}}=\varepsilon_{\mathrm{fx}}-\left.\varepsilon_{\mathrm{cx}}\right|_{\mathrm{Z}= \pm \mathrm{t}_{\mathrm{c}}}$

$\frac{\partial \Delta_{\mathrm{y}}}{\partial \mathrm{y}}=\varepsilon_{\mathrm{fy}}-\left.\varepsilon_{\mathrm{cy}}\right|_{\mathrm{z}= \pm \mathrm{t}_{\mathrm{c}}}$

$\gamma_{\mathrm{fxy}}=\left.\gamma_{\mathrm{cxy}}\right|_{\mathrm{Z}= \pm \mathrm{t}_{\mathrm{c}}}$

in which

Eand $\quad=$ Normal and shear strain, respectively;

$\Delta_{\mathrm{i}} \quad=$ Interlayer deformation in the i direction, where $\mathrm{i}=\mathrm{x}$ or $\mathrm{y}$;

$=\frac{\mathrm{q}_{\mathrm{i}}}{\mathrm{K}_{\mathrm{i}}} ;$

$\mathrm{K}_{\mathrm{i}} \quad=$ Stiffness of adhesive in the i direction.

Solutions to the problem must also satisfy the prescribed displacement boundary conditions. With respect to a panel subjected to edgewise loads, the relevant boundary conditions are

1. At the panel edges, no normal or shear stresses should exist in the fluteand the liner normal stress must equal the applied inplane stress, thus
at $\mathrm{x}=0,2 \mathrm{a}$
$\sigma_{\mathrm{fx}}=\sigma_{\mathrm{fxo}}$
$\sigma_{\mathrm{cx}}=\sigma_{\mathrm{cxo}}$
at $\mathrm{y}=0,2 \mathrm{~b}$
$\sigma_{\mathrm{fy}}=\sigma_{\mathrm{fyo}}$
$\sigma_{\mathrm{cy}}=\sigma_{\mathrm{cyo}}$

in which

$\begin{array}{ll}\sigma_{\text {fxo }} & =p_{\text {fxo }} / t_{f} \\ \sigma_{\text {fyo }} & =p_{\text {fyo }} / t_{f}\end{array}$

2. For symmetrical loading about the panel middle plane and centerlines, the shear stresses vanish and no in-plane displacements occur. Thus,
at $\mathrm{x}=\mathrm{a}$
$\tau_{\mathrm{fxy}}=\tau_{\mathrm{cxy}}=0$
$\mathrm{u}_{\mathrm{c}}=\mathrm{u}_{\mathrm{f}}=0$
at $\mathrm{y}=\mathrm{b}$
$\tau_{\mathrm{fyx}}=\tau_{\mathrm{cyx}}=0$
$\mathrm{v}_{\mathrm{c}}=\mathrm{v}_{\mathrm{f}}=0$

where $\mathrm{u}$ and $\mathrm{v}$ are displacements in the $\mathrm{x}$ and $\mathrm{y}$ directions, respectively.

\section{Equilibrium of Flute}

For the panel in Figure2, a solution for normal stress components in the flutesatisfying the boundary conditions in Equations 12 and 13 is considered as $(14,15,16,17,18)$

$$
\sigma_{\mathrm{cx}}=\sum_{\mathrm{m}=1,3, . .}^{\infty} \sum_{\mathrm{n}=1,3, . .}^{\infty} \mathrm{A}_{\mathrm{mn}} \phi_{\mathrm{x}} \mathrm{S}_{\mathrm{x}} \mathrm{S}_{y}+\sigma_{\mathrm{cxo}}
$$


$\sigma_{\text {cy }}=\sum_{\mathrm{m}=1,3, . .}^{\infty} \sum_{\mathrm{n}=1,3, . .}^{\infty} \mathrm{C}_{\mathrm{mn}} \phi_{\mathrm{y}} \mathrm{S}_{\mathrm{x}} \mathrm{S}_{y}+\sigma_{\text {cyo }}$

in which

$\sigma_{\mathrm{cxo}}, \sigma_{\mathrm{cyo}} \quad=$ Edge stresses in the flutein the $\mathrm{x}$ and $\mathrm{y}$ directions, respectively;

$\phi_{\mathrm{x}}=\theta_{\mathrm{x}}\left(2 \theta_{\mathrm{x}} \cosh \theta_{\mathrm{x}} \mathrm{z}+\mathrm{z} \theta_{\mathrm{x}}^{2} \sinh \theta_{\mathrm{x}} \mathrm{z}-\mathrm{t}_{\mathrm{c}} \theta_{\mathrm{x}}^{2} \operatorname{coth} \theta_{\mathrm{x}} \mathrm{t}_{\mathrm{c}} \cosh \theta_{\mathrm{x}} \mathrm{z}\right)-\frac{\alpha_{\mathrm{m}}^{2}}{2} \mathrm{k}_{\phi x} \cos \alpha_{\phi x} \mathrm{z}$

$\phi_{\mathrm{y}}=\theta_{\mathrm{y}}\left(2 \theta_{\mathrm{y}} \cosh \theta_{\mathrm{y}} \mathrm{z}+\mathrm{z} \theta_{\mathrm{y}}^{2} \sinh \theta_{\mathrm{y}} \mathrm{z}-\mathrm{t}_{\mathrm{c}} \theta_{\mathrm{y}}^{2} \operatorname{coth} \theta_{\mathrm{y}} \mathrm{t}_{\mathrm{c}} \cosh \theta_{\mathrm{y}} \mathrm{z}\right)-\frac{\beta_{\mathrm{n}}^{2}}{2} \mathrm{k}_{\phi \mathrm{y}} \cos \alpha_{\phi \mathrm{y}} \mathrm{z}$

$\mathrm{k}_{\phi x}=-\frac{\theta_{\mathrm{x}} \mathrm{t}_{\mathrm{c}}}{\sinh \theta_{\mathrm{x}} \mathrm{t}_{\mathrm{c}}}+\theta_{\mathrm{x}} \mathrm{t}_{\mathrm{c}} \cosh \theta_{\mathrm{x}} \mathrm{t}_{\mathrm{c}}$

$\mathrm{k}_{\phi y}=-\frac{\theta_{\mathrm{y}} \mathrm{t}_{\mathrm{c}}}{\sinh \theta_{\mathrm{y}} \mathrm{t}_{\mathrm{c}}}+\theta_{\mathrm{y}} \mathrm{t}_{\mathrm{c}} \cosh \theta_{\mathrm{y}} \mathrm{t}_{\mathrm{c}}$

$\theta_{\mathrm{x}}=\alpha_{\mathrm{m}} \sqrt{\frac{\mathrm{E}_{\mathrm{cx}}}{\mathrm{G}_{\mathrm{cxz}}}}$

$\theta_{\mathrm{y}}=\beta_{\mathrm{n}} \sqrt{\frac{\mathrm{E}_{\mathrm{cy}}}{\mathrm{G}_{\mathrm{cyz}}}}$

$\begin{array}{ll}\alpha_{\phi x} & =\frac{m \pi}{2 t_{c}} \\ \beta_{\phi y} & =\frac{n \pi}{2 t_{c}}\end{array}$

$\mathrm{S}_{\mathrm{x}}, \mathrm{S}_{\mathrm{y}} \quad=\sin \alpha_{\mathrm{m}} \mathrm{x}$ and $\sin \beta_{\mathrm{n}} \mathrm{y}$, respectively

$\alpha_{\mathrm{m}}, \beta_{\mathrm{n}} \quad=\frac{\mathrm{m} \pi}{2 \mathrm{a}}$ and $\frac{\mathrm{n} \pi}{2 \mathrm{~b}}$, respectively

$\mathrm{A}_{\mathrm{mn}}, \mathrm{C}_{\mathrm{mn}} \quad=\quad$ Unknown coefficients

$\mathrm{m}, \mathrm{n} \quad=$ Integers.

UsingEquations 16 and 17, expressions for the displacement of the flutethat satisfy the boundary conditions in Equations 14 and 15 are derived as follow

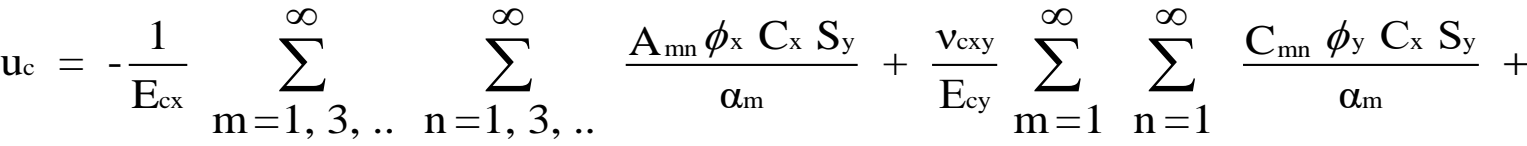

$$
\begin{aligned}
& (x-a)\left(\frac{\sigma_{c x o}}{E_{c x}}-\frac{\nu_{c x y} \sigma_{c y o}}{E_{c y}}\right)
\end{aligned}
$$




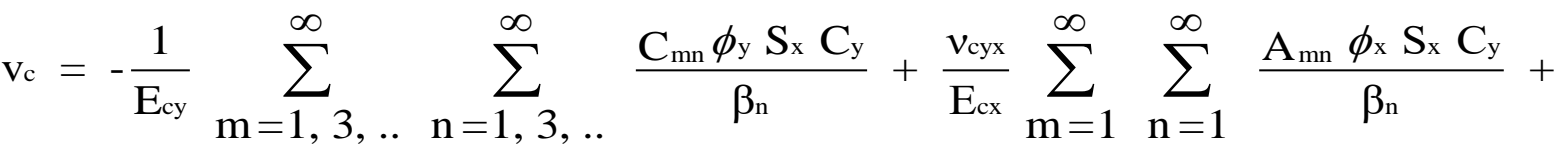

$$
\begin{aligned}
& (y-b)\left(\frac{\sigma_{c y o}}{E_{c y}}-\frac{\nu_{c x y} \sigma_{c x o}}{E_{c x}}\right)
\end{aligned}
$$

in which

$v_{\mathrm{cxy}}, v_{\mathrm{cyx}} \quad=$ Poisson's ratio in the $\mathrm{x}$ plane and y-direction, and the y-plane and $\mathrm{x}$-direction, respectively.

$\mathrm{C}_{\mathrm{x}}, \mathrm{C}_{\mathrm{y}} \quad=\cos \alpha_{\mathrm{m}} \mathrm{x}$ and $\cos \beta_{\mathrm{n}} \mathrm{y}$, respectively.

Equations 18 and 19 fulfill the boundary conditions in Equations 14 and 15. An expression for the shear strain in the flute $\gamma_{c x y}$ is obtained by properly differentiating Equations 18 and 19; thus

$$
\begin{aligned}
& \gamma_{\mathrm{cxy}}= \frac{1}{\mathrm{E}_{\mathrm{cx}}} \sum_{\mathrm{m}=1,3, . .}^{\infty} \sum_{\mathrm{n}=1,3, . .}^{\infty} \mathrm{A}_{\mathrm{mn}} \phi_{\mathrm{x}}\left(-\frac{\beta_{\mathrm{n}}}{\alpha_{\mathrm{m}}}+v_{\mathrm{cyx}} \frac{\alpha_{\mathrm{m}}}{\beta_{\mathrm{n}}}\right) \mathrm{C}_{\mathrm{x}} \mathrm{C}_{\mathrm{y}}+ \\
& \frac{1}{\mathrm{E}_{\mathrm{cy}}} \sum_{\mathrm{m}=1,3, . . \quad \mathrm{n}=1,3, . .}^{\infty} \mathrm{C}_{\mathrm{mn}} \phi_{\mathrm{y}}\left(-\frac{\alpha_{\mathrm{m}}}{\beta_{\mathrm{n}}}+v_{\mathrm{cxy}} \frac{\beta_{\mathrm{n}}}{\alpha_{\mathrm{m}}}\right) \mathrm{C}_{\mathrm{x}} \mathrm{C}_{\mathrm{y}}
\end{aligned}
$$

Equation 20 fulfills the boundary conditions in Equations 14 and 15. By substituting Equations 16, 17 and 20 in Equation 3, an expression for the vertical shear stress $\tau_{\mathrm{cxz}}$ in the fluteis obtained as

$$
\begin{gathered}
\tau_{\mathrm{cxz}}=\sum_{\mathrm{m}=1,3, . . \mathrm{n}=1,3, . .}^{\infty} \sum_{\mathrm{z}=0}^{\infty} \phi_{\mathrm{x}} \mathrm{dz} \mathrm{A}_{\mathrm{mn}}\left[-\alpha_{\mathrm{m}}+\frac{\mathrm{G}_{\mathrm{cxy}}}{\mathrm{E}_{\mathrm{cx}}} \beta_{\mathrm{n}}\left(-\frac{\beta_{\mathrm{n}}}{\alpha_{\mathrm{m}}}+v_{\mathrm{cyx}} \frac{\alpha_{\mathrm{m}}}{\beta_{\mathrm{n}}}\right)\right] \mathrm{C}_{\mathrm{x}} \mathrm{S}_{\mathrm{y}}+ \\
\frac{\mathrm{G}_{\mathrm{cxy}}}{\mathrm{E}_{\mathrm{cy}}} \sum_{\mathrm{m}=1,3, . . \mathrm{n}=1,3, . .}^{\infty} \int_{\mathrm{z}=0}^{\infty} \phi_{\mathrm{y}} \mathrm{dzC_{ \textrm {mn } }} \beta_{\mathrm{n}}\left(-\frac{\alpha_{\mathrm{m}}}{\beta_{\mathrm{n}}}+v_{\mathrm{cxy}} \frac{\beta_{\mathrm{n}}}{\alpha_{\mathrm{m}}}\right) \mathrm{C}_{\mathrm{x}} \mathrm{S}_{\mathrm{y}}
\end{gathered}
$$

In a similar manner, the shear stress in the flute $\tau_{\text {cyz }}$ is obtained from Equations 17, 20 and 4 as

$$
\begin{gathered}
\tau_{\mathrm{cyz}}=\sum_{\mathrm{m}=1,3, . . \mathrm{n}=1,3, . .}^{\infty} \sum_{\mathrm{z}=0}^{\infty} \phi_{\mathrm{y}} \mathrm{dz} \mathrm{C}_{\mathrm{mn}}\left[-\beta_{\mathrm{m}}+\frac{\mathrm{G}_{\mathrm{cxy}}}{\mathrm{E}_{\mathrm{cy}}} \alpha_{\mathrm{m}}\left(-\frac{\alpha_{\mathrm{m}}}{\beta_{\mathrm{n}}}+v_{\mathrm{cxy}} \frac{\beta_{\mathrm{n}}}{\alpha_{\mathrm{m}}}\right)\right] \mathrm{S}_{\mathrm{x}} \mathrm{C}_{\mathrm{y}}+ \\
\frac{\mathrm{G}_{\mathrm{cxy}}}{\mathrm{E}_{\mathrm{cx}}} \sum_{\mathrm{m}=1,3, . . \mathrm{n}=1,3, . .}^{\infty} \int_{\mathrm{z}=0}^{\infty} \phi_{\mathrm{x}} \mathrm{dz} \mathrm{A}_{\mathrm{mn}} \alpha_{\mathrm{m}}\left(-\frac{\beta_{\mathrm{n}}}{\alpha_{\mathrm{m}}}+v_{\mathrm{cyx}} \frac{\alpha_{\mathrm{m}}}{\beta_{\mathrm{n}}}\right) \mathrm{S}_{\mathrm{x}} \mathrm{C}_{\mathrm{y}}
\end{gathered}
$$

\section{Interlayer Shear Stresses}

Expressions for the interlayer shear stresses $\mathrm{q}_{\mathrm{x}}$ and $\mathrm{q}_{\mathrm{y}}$ are obtained from Equations 21 and 22 in accordance with the compatibility equations 7 and 8; thus

$$
\left.\mathrm{q}_{\mathrm{x}}=\sum_{\mathrm{m}=1,3, . . \mathrm{n}=1,3, . .}^{\infty} \sum_{\mathrm{mn}}^{\infty} \lambda_{\mathrm{gn} 1}+\mathrm{C}_{\mathrm{mn}} \lambda_{\mathrm{gn} 2}\right) \mathrm{C}_{\mathrm{x}} \mathrm{S}_{\mathrm{y}}
$$


$\left.\mathrm{q}_{\mathrm{y}}=\sum_{\mathrm{m}=1,3, . . \mathrm{n}=1,3, . .}^{\infty} \sum_{\mathrm{mn}}^{\infty} \lambda_{\mathrm{gk} 1}+\mathrm{A}_{\mathrm{mn}} \lambda_{\mathrm{gk} 2}\right) \mathrm{S}_{\mathrm{x}} \mathrm{C}_{\mathrm{y}}$

in which

$$
\begin{aligned}
& \lambda_{\mathrm{gn} 1}=\left.\int_{\mathrm{z}=0}^{\mathrm{z}} \phi_{\mathrm{x}} \mathrm{dz}\right|_{\mathrm{Z}=\mathrm{t}_{\mathrm{c}}}\left[-\alpha_{\mathrm{m}}+\frac{\mathrm{G}_{\mathrm{cxy}}}{\mathrm{E}_{\mathrm{cx}}} \beta_{\mathrm{n}}\left(-\frac{\beta_{\mathrm{n}}}{\alpha_{\mathrm{m}}}+v_{\mathrm{cyx}} \frac{\alpha_{\mathrm{m}}}{\beta_{\mathrm{n}}}\right)\right] \\
& \lambda_{\mathrm{gn} 2}=\left.\frac{\mathrm{G}_{\mathrm{cxy}}}{\mathrm{E}_{\mathrm{cy}}} \int_{\mathrm{z}=0}^{\mathrm{z}} \phi_{\mathrm{y}} \mathrm{dz}\right|_{\mathrm{Z}=\mathrm{t}_{\mathrm{c}}} \beta_{\mathrm{n}}\left(-\frac{\alpha_{\mathrm{m}}}{\beta_{\mathrm{n}}}+v_{\mathrm{cxy}} \frac{\beta_{\mathrm{n}}}{\alpha_{\mathrm{m}}}\right) \\
& \lambda_{\mathrm{gk} 1}=\left.\int_{\mathrm{z}=0}^{\mathrm{z}} \phi_{\mathrm{y}} \mathrm{dz}\right|_{\mathrm{z}=\mathrm{t}_{\mathrm{c}}}\left[-\beta_{\mathrm{n}}+\frac{\mathrm{G}_{\mathrm{cxy}}}{\mathrm{E}_{\mathrm{cy}}} \alpha_{\mathrm{m}}\left(-\frac{\alpha_{\mathrm{m}}}{\beta_{\mathrm{n}}}+v_{\mathrm{cxy}} \frac{\beta_{\mathrm{n}}}{\alpha_{\mathrm{m}}}\right)\right] \\
& \lambda_{\mathrm{gk} 2}=\left.\frac{\mathrm{G}_{\mathrm{cxy}}}{\mathrm{E}_{\mathrm{cx}}} \int_{\mathrm{z}=0}^{\mathrm{z}} \phi_{\mathrm{x}} \mathrm{dz}\right|_{\mathrm{z}=\mathrm{t}_{\mathrm{c}}} \alpha_{\mathrm{m}}\left(-\frac{\beta_{\mathrm{n}}}{\alpha_{\mathrm{m}}}+v_{\mathrm{cyx}} \frac{\alpha_{\mathrm{m}}}{\beta_{\mathrm{n}}}\right)
\end{aligned}
$$

\section{Equilibrium of Liner}

An expression for the in-plane shear stress $\tau_{\mathrm{fxy}}$ in the liners is obtained from Equations 11 and 20 as

$$
\begin{array}{r}
\tau_{\mathrm{fxy}}=\sum_{\mathrm{m}=1,3, \ldots} \sum_{\mathrm{n}=1,3, . .}^{\infty} \mathrm{A}_{\mathrm{mn}}\left[\left.\frac{\mathrm{G}_{\mathrm{fxy}}}{\mathrm{E}_{\mathrm{cx}}} \phi_{\mathrm{x}}\right|_{\mathrm{z}=\mathrm{tc}}\left(-\frac{\beta_{\mathrm{n}}}{\alpha_{\mathrm{m}}}+v_{\mathrm{cyx}} \frac{\alpha_{\mathrm{m}}}{\beta_{\mathrm{n}}}\right)+\right. \\
\left.\frac{\mathrm{G}_{\mathrm{fxy}} \beta_{\mathrm{n}}}{\mathrm{K}_{\mathrm{x}}} \lambda_{\mathrm{gn} 1}+\frac{\mathrm{G}_{\mathrm{fxy}} \boldsymbol{\alpha}_{\mathrm{m}}}{\mathrm{K}_{\mathrm{y}}} \lambda_{\mathrm{gk} 2}\right] \mathrm{C}_{\mathrm{x}} \mathrm{C}_{\mathrm{y}}+ \\
\sum_{\mathrm{m}=1,3, . .}^{\infty} \sum_{\mathrm{n}=1,3, . .}^{\infty} \mathrm{C}_{\mathrm{mn}}\left[\left.\frac{\mathrm{G}_{\mathrm{fxy}}}{\mathrm{E}_{\mathrm{cy}}} \phi_{\mathrm{y}}\right|_{\mathrm{z}=\mathrm{tc}}\left(-\frac{\alpha_{\mathrm{m}}}{\beta_{\mathrm{n}}}+v_{\mathrm{cxy}} \frac{\beta_{\mathrm{n}}}{\alpha_{\mathrm{m}}}\right)+\right. \\
\left.\frac{\mathrm{G}_{\mathrm{fxy}} \beta_{\mathrm{n}}}{\mathrm{K}_{\mathrm{x}}} \lambda_{\mathrm{gn} 2}+\frac{\mathrm{G}_{\mathrm{fxy}} \boldsymbol{\alpha}_{\mathrm{m}}}{\mathrm{K}_{\mathrm{y}}} \lambda_{\mathrm{gk} 1}\right] \mathrm{C}_{\mathrm{x}} \mathrm{C}_{\mathrm{y}}
\end{array}
$$

An expression for the normal stress $\sigma_{\mathrm{fx}}$ in the liners is obtained by substituting Equations 23 and 25 in Equation 1; Thus

$$
\sigma_{\mathrm{fx}}=\sum_{\mathrm{m}=1,3, . . \mathrm{n}=1,3, . .}^{\infty}\left(\mathrm{A}_{\mathrm{mn}} \lambda_{\mathrm{z} 1}+\mathrm{C}_{\mathrm{mn}} \lambda_{\mathrm{z} 2}\right) \mathrm{S}_{\mathrm{x}} \mathrm{S}_{\mathrm{y}}+\mathrm{f}(\mathrm{x}, \mathrm{y})
$$

in which

$\lambda_{\mathrm{z} 1}=\left.\frac{\mathrm{G}_{\mathrm{fxy}}}{\mathrm{E}_{\mathrm{cx}}} \phi_{\mathrm{x}}\right|_{\mathrm{Z}=\mathrm{t}_{\mathrm{c}}} \frac{\beta_{\mathrm{n}}}{\alpha_{\mathrm{m}}}\left(-\frac{\beta_{\mathrm{n}}}{\alpha_{\mathrm{m}}}+v_{\mathrm{cyx}} \frac{\alpha_{\mathrm{m}}}{\beta_{\mathrm{n}}}\right)+\frac{\lambda_{\mathrm{gn} 1}}{\alpha_{\mathrm{m}}}\left(\frac{\mathrm{G}_{\mathrm{fxy}} \beta_{\mathrm{n}}^{2}}{\mathrm{~K}_{\mathrm{x}}}+\frac{1}{\mathrm{t}_{\mathrm{f}}}\right)+\frac{G_{f x y} \lambda_{g k 2} \beta_{n}}{K_{y}}$ 
$\lambda_{\mathrm{z} 2}=\left.\frac{\mathrm{G}_{\mathrm{fxy}}}{\mathrm{E}_{\mathrm{cy}}} \phi_{\mathrm{y}}\right|_{\mathrm{Z}=\mathrm{t}_{\mathrm{c}}} \frac{\beta_{\mathrm{n}}}{\alpha_{\mathrm{m}}}\left(-\frac{\alpha_{\mathrm{m}}}{\beta_{\mathrm{n}}}+v_{\mathrm{cxy}} \frac{\beta_{\mathrm{n}}}{\alpha_{\mathrm{m}}}\right)+\frac{\lambda_{\mathrm{gn} 2}}{\alpha_{\mathrm{m}}}\left(\frac{\mathrm{G}_{\mathrm{fxy}} \beta_{\mathrm{n}}^{2}}{\mathrm{~K}_{\mathrm{x}}}+\frac{1}{\mathrm{t}_{\mathrm{f}}}\right)+\frac{\mathrm{G}_{\mathrm{fxy}} \lambda_{\mathrm{gk} 1} \beta_{\mathrm{n}}}{\mathrm{K}_{\mathrm{y}}}$

$\mathrm{f}(\mathrm{x}, \mathrm{y}) \quad=\quad$ A function representing the constant of integration.

The function $\mathrm{f}(\mathrm{x}, \mathrm{y})$ is obtained by using the overall equilibrium equation 5; Equations 16 and 26, and by expanding the applied load in double trigonometric series. In the case of a uniform load of intensities $\mathrm{p}_{\mathrm{fxo}}$ and $\mathrm{p}_{\mathrm{cxo}}, \sigma_{\mathrm{fx}}$ is found as

$$
\sigma_{\mathrm{fx}}=\sum_{\mathrm{m}=1,3, . .}^{\infty} \sum_{\mathrm{n}=1,3, . .}^{\infty}\left[\mathrm{A}_{\mathrm{mn}} \lambda_{\mathrm{z} 1}^{\prime}+\mathrm{C}_{\mathrm{mn}} \lambda_{\mathrm{z} 2}^{\prime}\right] \mathrm{S}_{\mathrm{x}} \mathrm{S}_{\mathrm{y}}+\left(\sigma_{\mathrm{xo}}+\sigma_{\mathrm{cxo}} \frac{\mathrm{t}_{\mathrm{c}}}{\mathrm{t}_{\mathrm{f}}}\right) \sum_{\mathrm{m}=1}^{\infty} \sum_{\mathrm{n}=1}^{\infty} \frac{2}{\mathrm{a} \alpha_{\mathrm{m}}} \frac{2}{\mathrm{~b} \beta_{\mathrm{n}}} \mathrm{S}_{\mathrm{x}} \mathrm{S}_{\mathrm{y}}
$$

in which

$$
\begin{aligned}
& \lambda_{\mathrm{z} 1}^{\prime}=\lambda_{\mathrm{z} 1}\left(1-\frac{2}{\mathrm{~b}^{2} \beta_{\mathrm{n}}^{2}}\right)-\frac{2 \int_{0}^{\mathrm{tc}} \phi_{\mathrm{x}} \mathrm{dz}}{\mathrm{tf} \mathrm{b}^{2} \beta_{\mathrm{n}}^{2}} \\
& \lambda_{\mathrm{z} 2}^{\prime}=\lambda_{\mathrm{z} 2}\left(1-\frac{2}{\mathrm{~b}^{2} \beta_{\mathrm{n}}^{2}}\right)
\end{aligned}
$$

In a similar manner, an expression for the normal stress $\sigma_{\text {fy }}$ is obtained from Equations 2, 6, 17 and 24 as

$$
\left.\sigma_{\mathrm{fy}}=\sum_{\mathrm{m}=1,3, . . \mathrm{n}=1,3, . .}^{\infty} \sum_{\mathrm{Amn}}^{\infty} \lambda_{\mathrm{z} 3}^{\prime}+\mathrm{C}_{\mathrm{mn}} \lambda_{\mathrm{z} 4}^{\prime}\right] \mathrm{S}_{\mathrm{x}} \mathrm{S}_{\mathrm{y}}+\left(\sigma_{\mathrm{yo}}+\sigma_{\mathrm{cyo}} \frac{\mathrm{t}_{\mathrm{c}}}{\mathrm{t}_{\mathrm{f}}}\right) \sum_{\mathrm{m}=1}^{\infty} \sum_{\mathrm{n}=1}^{\infty} \frac{2}{\mathrm{a} \alpha_{\mathrm{m}}} \frac{2}{\mathrm{~b} \beta_{\mathrm{n}}} \mathrm{S}_{\mathrm{x}} \mathrm{S}_{\mathrm{y}}
$$

in which

$$
\lambda_{\mathrm{z} 3}^{\prime}=\lambda_{\mathrm{z} 3}\left(1-\frac{2}{\mathrm{a}^{2} \alpha_{\mathrm{m}}^{2}}\right)
$$

$\lambda_{\mathrm{z} 4}^{\prime}=\lambda_{\mathrm{z} 4}\left(1-\frac{2}{\mathrm{a}^{2} \alpha_{\mathrm{n}}^{2}}\right)-\frac{2 \int_{0}^{\mathrm{t}_{\mathrm{c}}} \phi_{\mathrm{y}} \mathrm{dz}}{\mathrm{tf}_{\mathrm{f}} \mathrm{a}^{2} \alpha_{\mathrm{m}}^{2}}$

$\lambda_{\mathrm{z} 3}=\left.\frac{\mathrm{G}_{\mathrm{fxy}}}{\mathrm{E}_{\mathrm{cx}}} \phi_{\mathrm{x}}\right|_{\mathrm{Z}=\mathrm{t}_{\mathrm{c}}} \frac{\alpha_{\mathrm{m}}}{\beta_{\mathrm{n}}}\left(-\frac{\beta_{\mathrm{n}}}{\alpha_{\mathrm{m}}}+v_{\mathrm{cyx}} \frac{\alpha_{\mathrm{m}}}{\beta_{\mathrm{n}}}\right)+\frac{\lambda_{\mathrm{gk} 2}}{\beta_{\mathrm{n}}}\left(\frac{\mathrm{G}_{\mathrm{fxy}} \alpha_{\mathrm{m}}^{2}}{\mathrm{~K}_{\mathrm{y}}}+\frac{1}{\mathrm{t}_{\mathrm{f}}}\right)+\frac{\mathrm{G}_{\mathrm{fxy}} \lambda_{\mathrm{gn} 1} \alpha_{\mathrm{m}}}{\mathrm{K}_{\mathrm{x}}}$

$\lambda_{\mathrm{z} 4}=\left.\frac{\mathrm{G}_{\mathrm{fxy}}}{\mathrm{E}_{\mathrm{cy}}} \phi_{\mathrm{y}}\right|_{\mathrm{Z}=\mathrm{t}_{\mathrm{c}}} \frac{\alpha_{\mathrm{m}}}{\beta_{\mathrm{n}}}\left(-\frac{\alpha_{\mathrm{m}}}{\beta_{\mathrm{n}}}+v_{\mathrm{cxy}} \frac{\beta_{\mathrm{n}}}{\alpha_{\mathrm{m}}}\right)+\frac{\lambda_{\mathrm{gk} 1}}{\beta_{\mathrm{n}}}\left(\frac{\mathrm{G}_{\mathrm{fxy}} \alpha_{\mathrm{n}}^{2}}{\mathrm{~K}_{\mathrm{y}}}+\frac{1}{\mathrm{t}_{\mathrm{f}}}\right)+\frac{\mathrm{G}_{\mathrm{fxy}} \lambda_{\mathrm{gn} 2} \alpha_{\mathrm{m}}}{\mathrm{K}_{\mathrm{x}}}$

Solutions for $\mathrm{A}_{\mathrm{mn}}$ and $\mathrm{C}_{\mathrm{mn}}$

At this stage, the only unknowns are the coefficients $A_{m n}$ and $C_{m n}$. These can be determined by using the compatibility equations 9 and 10. By substituting Equations 16, 17, 23, 24, 27 and 28 in Equations 9 and 10; $\mathrm{A}_{\mathrm{mn}}$ and $\mathrm{C}_{\mathrm{mn}}$ are obtained as 


$$
\begin{array}{r}
\mathrm{A}_{\mathrm{mn}}=\frac{\frac{\lambda_{\mathrm{y} 3}}{\lambda_{\mathrm{y} 2}}-\frac{\lambda_{\mathrm{y} 6}}{\lambda_{\mathrm{y} 5}}}{\frac{\lambda_{\mathrm{y} 1}}{\lambda_{\mathrm{y} 2}}-\frac{\lambda_{\mathrm{y} 4}}{\lambda_{\mathrm{y} 5}}} \\
\mathrm{C}_{\mathrm{mn}}=\frac{\frac{\lambda_{\mathrm{y} 3}}{\lambda_{\mathrm{y} 1}}-\frac{\lambda_{\mathrm{y} 6}}{\lambda_{\mathrm{y} 4}}}{\frac{\lambda_{\mathrm{y} 2}}{\lambda_{\mathrm{y} 1}}-\frac{\lambda_{\mathrm{y} 5}}{\lambda_{\mathrm{y} 4}}}
\end{array}
$$

in which

$\lambda_{\mathrm{y} 1}=\frac{\lambda_{\mathrm{z} 1}^{\prime}}{\mathrm{E}_{\mathrm{fx}}}-\frac{v_{\mathrm{fxy}} \lambda_{\mathrm{z} 3}^{\prime}}{\mathrm{E}_{\mathrm{fy}}}-\frac{\left.\phi_{\mathrm{x}}\right|_{\mathrm{z}=\mathrm{tc}}}{\mathrm{E}_{\mathrm{cx}}}+\frac{\alpha_{\mathrm{m}} \lambda_{\mathrm{gn} 1}}{\mathrm{~K}_{\mathrm{x}}}$

$\lambda_{\mathrm{y} 2}=\frac{\lambda_{\mathrm{z} 2}^{\prime}}{\mathrm{E}_{\mathrm{fx}}}-\frac{\nu_{\mathrm{fxy}} \lambda_{\mathrm{z} 4}^{\prime}}{\mathrm{E}_{\mathrm{fy}}}+\frac{\left.\nu_{\mathrm{cxy}} \phi_{\mathrm{y}}\right|_{\mathrm{z}=\mathrm{tc}}}{\mathrm{E}_{\mathrm{cy}}}+\frac{\alpha_{\mathrm{m}} \lambda_{\mathrm{gn} 2}}{\mathrm{~K}_{\mathrm{x}}}$

$\lambda_{\mathrm{y} 3}=\frac{2}{\mathrm{a} \alpha_{\mathrm{m}}} \frac{2}{\mathrm{~b} \beta_{\mathrm{n}}}\left[\frac{\sigma_{\mathrm{xo}}+\sigma_{\mathrm{cxo}} \frac{\mathrm{t}_{\mathrm{c}}}{\mathrm{t}_{\mathrm{f}}}}{\mathrm{E}_{\mathrm{fx}}}-v_{\mathrm{fxy}} \frac{\sigma_{\mathrm{yo}}+\sigma_{\mathrm{cyo}} \frac{\mathrm{t}_{\mathrm{c}}}{\mathrm{t}_{\mathrm{f}}}}{\mathrm{E}_{\mathrm{fy}}}+\frac{\sigma_{\mathrm{cxo}}}{\mathrm{E}_{\mathrm{cx}}}+v_{\mathrm{cxy}} \frac{\sigma_{\mathrm{cyo}}}{\mathrm{E}_{\mathrm{cy}}}\right]$

$\lambda_{\mathrm{y} 4}=\frac{\lambda_{\mathrm{z} 3}^{\prime}}{E_{\mathrm{fy}}}-\frac{v_{\mathrm{fyx}} \lambda_{\mathrm{z} 1}^{\prime}}{E_{\mathrm{fx}}}+\frac{\left.\nu_{\mathrm{cyx}} \phi_{\mathrm{x}}\right|_{\mathrm{z}=\mathrm{tc}}}{E_{\mathrm{cx}}}+\frac{\beta_{\mathrm{n}} \lambda_{\mathrm{gk} 2}}{K_{\mathrm{y}}}$

$\lambda_{\mathrm{y} 5}=\frac{\lambda_{\mathrm{z} 4}^{\prime}}{E_{\mathrm{fy}}}-\frac{v_{\mathrm{fyx}} \lambda_{\mathrm{z} 2}^{\prime}}{E_{\mathrm{fx}}}-\frac{\left.\phi_{\mathrm{y}}\right|_{\mathrm{z}=\mathrm{tc}}}{E_{\mathrm{cy}}}+\frac{\beta_{\mathrm{n}} \lambda_{\mathrm{gk} 1}}{\mathrm{~K}_{\mathrm{y}}}$

$\lambda_{\mathrm{y} 6}=\frac{2}{\mathrm{a} \alpha_{\mathrm{m}}} \frac{2}{\mathrm{~b} \beta_{\mathrm{n}}}\left[\frac{\sigma_{\mathrm{yo}}+\sigma_{\mathrm{cyo}} \frac{\mathrm{t}_{\mathrm{c}}}{\mathrm{t}_{\mathrm{f}}}}{\mathrm{E}_{\mathrm{fy}}}-v_{\mathrm{fyx}} \frac{\sigma_{\mathrm{xo}}+\sigma_{\mathrm{cxo}} \frac{\mathrm{t}_{\mathrm{c}}}{\mathrm{t}_{\mathrm{f}}}}{\mathrm{E}_{\mathrm{fx}}}+\frac{\sigma_{\mathrm{cyo}}}{\mathrm{E}_{\mathrm{cy}}}+v_{\mathrm{cyx}} \frac{\sigma_{\mathrm{cxo}}}{\mathrm{E}_{\mathrm{cx}}}\right]$

\section{NUMERIC EVALUATION OF EFFECTS OF ADHESIVE ON THE BEHAVIOR OF PANELS}

The complexity of the preceding solution makes it difficult to see the effect of adhesive on the panel responses. To demonstrate these effects, a square panel is considered. The properties of the panel, liners and fluteare assumed as follow:

For panel:

$\mathrm{a}=\mathrm{b}$

$=20$ in. $(1219.2 \mathrm{~mm})$

For liners:

$\begin{array}{lll}\mathrm{t}_{\mathrm{f}} & =0.04 \mathrm{in} .(1.016 \mathrm{~mm}) ; \\ \mathrm{E}_{\mathrm{fx}}=\mathrm{E}_{\mathrm{fy}} & =10^{7} \mathrm{psi}(68.9 \mathrm{GPa}) ; \\ v_{\mathrm{fxy}}=v_{\mathrm{fyx}} & =0.33\end{array}$


For flute:

$t_{c}$

$\mathrm{E}_{\mathrm{cx}}=\mathrm{E}_{\mathrm{cy}}$

$\mathrm{G}_{\mathrm{cxy}}=\mathrm{G}_{\mathrm{cxz}}=\mathrm{G}_{\mathrm{cyz}}$

$v_{\mathrm{cxy}}=v_{\mathrm{cyx}}$
$=1.0 \mathrm{in} .(50.8 \mathrm{~mm})$

$=2 \times 10^{6} \mathrm{psi}(137.8 \mathrm{MPa})$

$=10^{4} \mathrm{psi}(68.9 \mathrm{MPa})$

$=0.20$

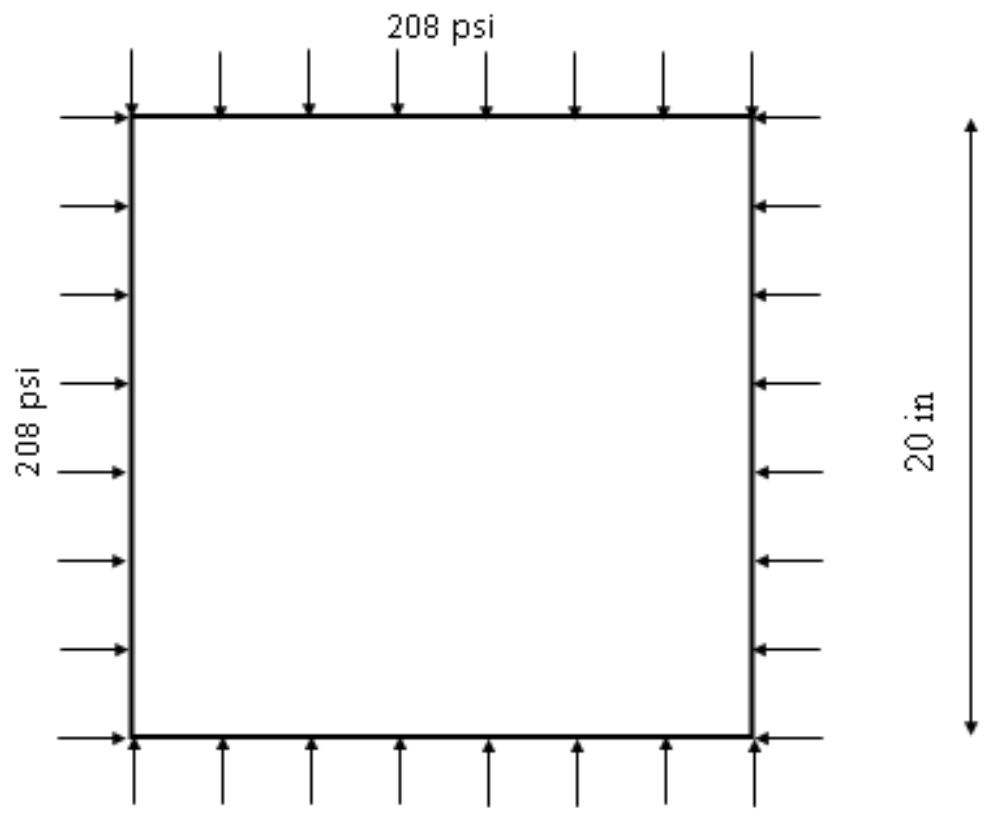

$20 \mathrm{in}$

Fig. 4a A Plane View of a Panel under Biaxial Edge Load

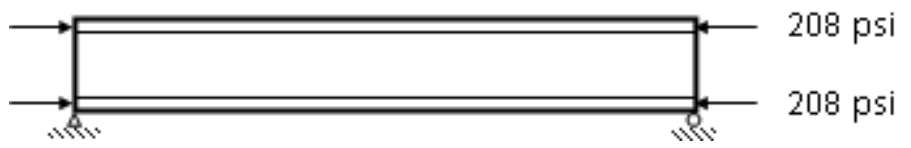

Fig. 4b A Biaxial Edge Load Applied to Faces

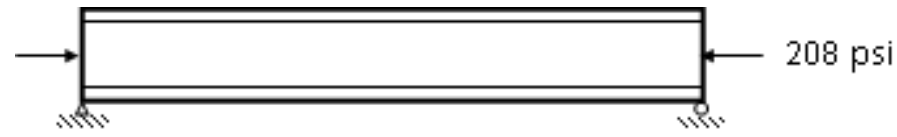

Fig. 4c A Biaxial Edge Load Applied to Core

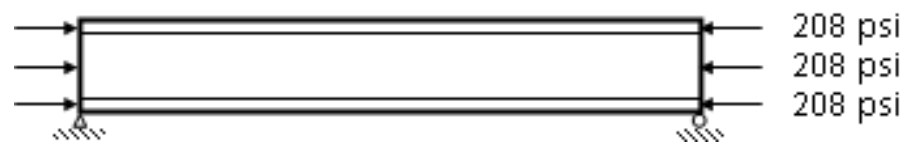

Fig. 4d A Biaxial Edge Load Applied to Faces and Core

Two loading cases are considered. In the first case a biaxial uniformly distributed stress of intensity $\sigma_{\mathrm{fxo}}=\sigma_{\mathrm{fyo}}=208.3 \mathrm{psi}$ is used. In the second case a uniaxial uniformly distributed stress of intensity $\sigma_{\mathrm{fxo}}=208.3 \mathrm{psi}$ is applied. In each loading case, the load is applied independently first to the liner and flute, and then concurrently to liner and flute as shown in Figures $4 \mathrm{a}, 4 \mathrm{~b}, 4 \mathrm{c}$ and $4 \mathrm{~d}$. The liner normal and shear stresses are calculated for a chosen range of bonding stiffness from $\mathrm{K}_{\mathrm{x}}=\mathrm{K}_{\mathrm{y}}=\mathrm{K}=10^{3}-10^{4} \mathrm{psi} / \mathrm{in}$. The selected range for $\mathrm{K}$-values covers a broad spectrum of adhesives from a non-rigid to excessively rigid for practical purposes. This range was needed for conducting a parametric study on the effects of adhesives. The normal stress in the liners at the panel center and the shear stress in the liners at the panel corner are shown graphically in Figures5 to 10. 


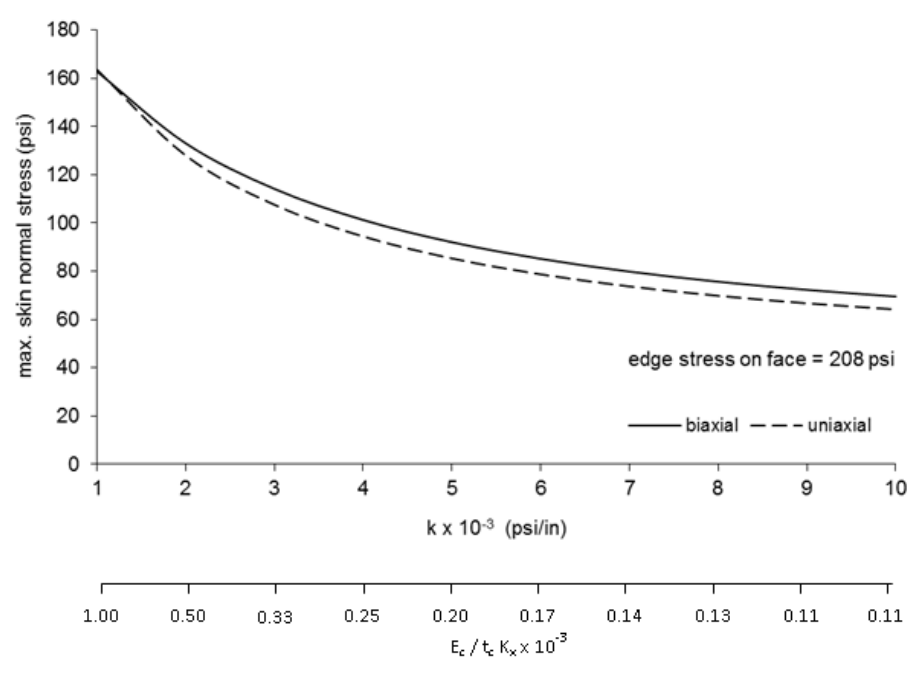

Fig. 5 Effects of Bond Stiffness on Face Normal Stress Due to Face Edge Load

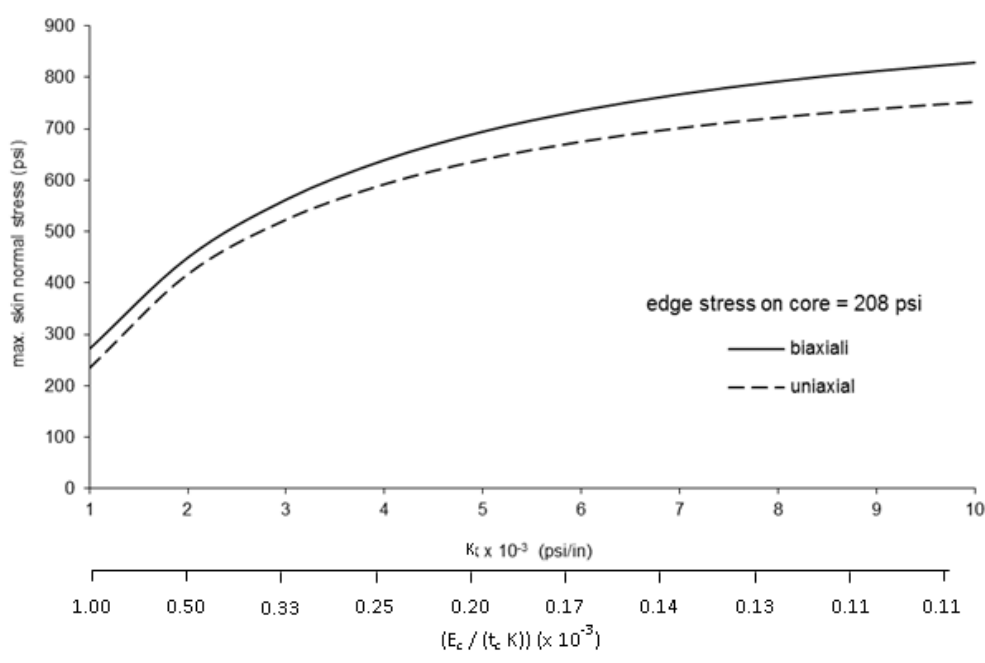

Fig. 6 Effects of Bond Stiffness on Face Normal Stress Due to Core Edge Load

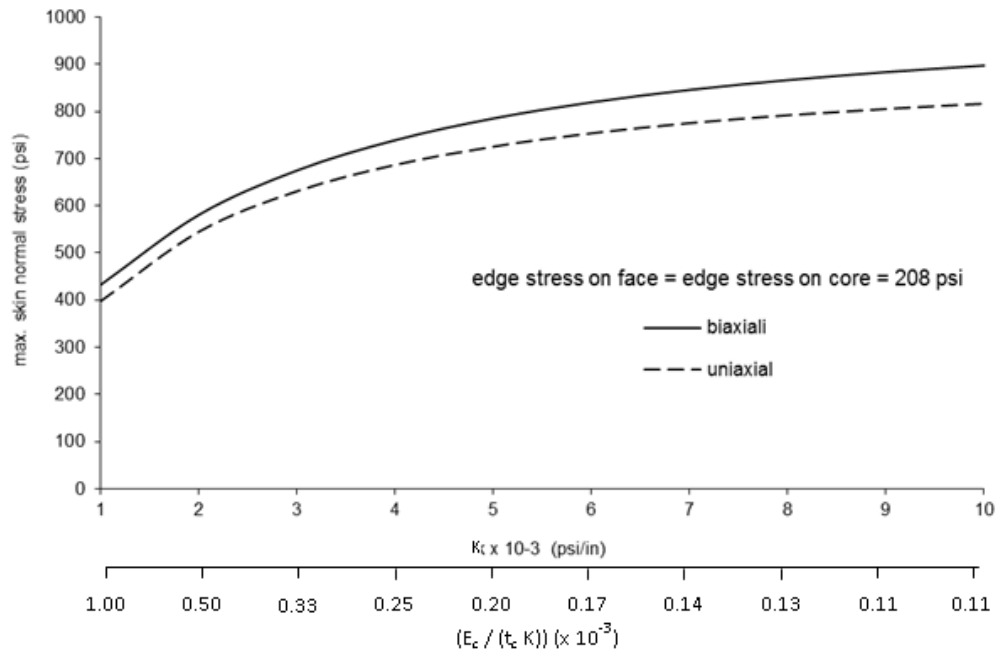

Fig. 7 Effects of Bond Stiffness on Face Normal Stress Due to Face and Core Edge Load 


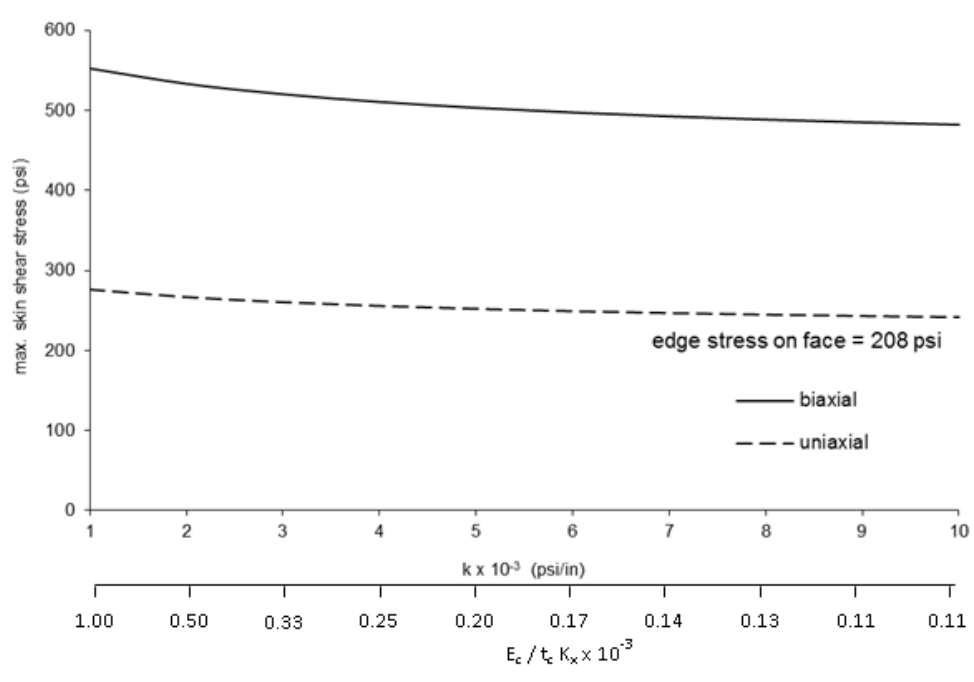

Fig. 8 Effects of Bond Stiffness on Face Shear Stress Due to Face Edge Load

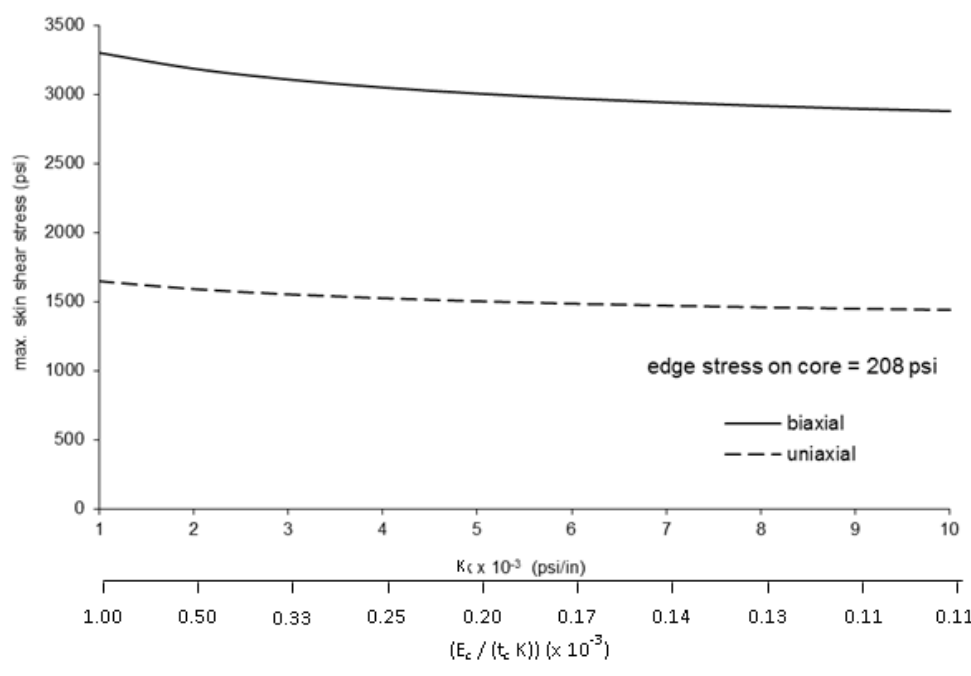

Fig. 9 Effects of Bond Stiffness on Face Shear Stress Due to Core Edge Load

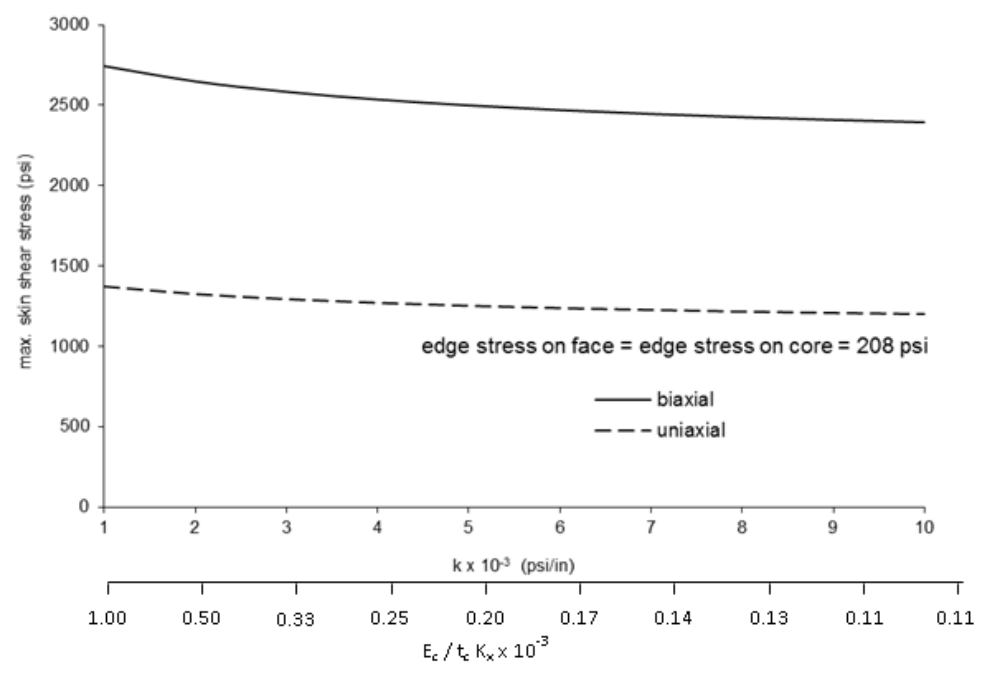

Fig. 10 Effects of Bond Stiffness on Face Shear Stress Due to Core Edge Load 
It is seen that the liner normal stress shows greater sensitivity to the variation of bond stiffness value when the latter is in the lower range; and beyond a certain level of stiffness (which varies from panel to panel), the adhesive can be practically considered as rigid. A change in K-value for example from $10^{3}$ to $2 \times 10^{3} \mathrm{psi} /$ in induces a stress decrease almost 6 times in the uniaxial case and 5 times in the biaxial case greater than when $\mathrm{K}$ changes from $9 \times 10^{3}-10^{4}$ psi/in. The changes are $24 \%$ and $27 \%$ due to uniaxial fluteand combined edge loads, respectively, $32 \%$ and $22 \%$ due to biaxial fluteand combined edge loads, respectively. In all load cases, the liner shear stress is practically independent of bonding stiffness. Unlike the mechanical behavior of other panels with non-rigid adhesive $(9,10,11,12,13,14,15,16$ and 18), this study reveals that interlayer shears are insignificant. This is due to the absence of transverse loads which induce high transverse shear forces.

This analysis has also detected an important point. By using existing theories (1, 24), stress components in panels may be determined only at high values of bond stiffness with a small margin of error, otherwise the $\mathrm{K}$ values must be included in the analysis.

Another important point was revealed by this analysis. By common sense, it can be felt that a very stiff adhesive would be unnecessary if the flute was too soft, and the converse would be unwise. This is quantitatively shown in Figures5 to 10 which show that the ratio of flute stiffness to bonding stiffness is one of the main parameters influencing the behavior of panels.

Finally, it is worthwhile to mention that the literature has no record of elasticity based analytic investigations of panelsassembled with adhesives having finite stiffness and under edgewise loads including the fluteedges. In this regard, this paper has advanced the state-of-the art. In addition, the results presented here are virtually identical to those by existing theories for the case of perfect; i.e. very rigid, adhesive.

\section{SUMMARY AND CONCLUSIONS}

In the literature, very few publicationsare availablethat deal with the effects of adhesives on the response of panels. Realistically, the adhesive in suchpanelsis rigid enough to make a significant contribution to the overall structural integrity of the panel, yet flexible enough to permit shear deformation.

An analysis of panels taking into account the effects of the finite bonding stiffness has been presented in this paper. The edgewise load can be uniaxial or biaxial; applied to the liners only, fluteonly, or to both components. The solution satisfies the equilibrium equations of the liner and flute, the compatibility equations of stresses and strains at the interfaces, and the boundary conditions.

The numerical results have shown that the bonding stiffness, up to a certain level, has a strong effect on the panel response. Beyond this level, the usual assumption of perfect bonding in the literature is quite acceptable. The answer to what constitute perfect bonding may be best answered in terms of the ratio of flutestiffnessto the bond stiffness, i.e. rather than on the individual constituent material. This ratio will vary from panel to another but could be determined using the solution presented in this paper.

\section{REFERENCES}

[1]. Allen, H. G. (1969). Analysis and Design of Structural Sandwich Panels. Pergamon Press Ltd.

[2]. Andrews, E. W. and Moussa, N. A. (2009). Failure mode maps for composite sandwich panels subjected to air blast loading. International Journal of Impact Engineering, Vol. 36.

[3]. Bujanovic, B., Personalcommunication, 2009.

[4]. Goodman, J. R., and Popov, E. P. (1968). Layered Beam Systems with Interlayer Slip. Journal of the Structural Engineering Division, ASCE, Vol. 94, No. ST11.

[5]. Goodman, J. R. (1969). Layered Wood Systems with Interlayer Slip. Wood Science, Vol. 1, No. 3.

[6]. Goodman, J. R., Vanderbilt, M. D., Criswell, M. E., and Bodig, J. (1974). Composite and Two-Way Action in Wood Joist Floor Systems," Wood Science, Vol. 7, No. 1.

[7]. Haan, D. L. (1996). A New Foam Core for Demanding Sandwich Structures. Proceedings of the Third International Conference on Sandwich Construction, Southampton UK.

[8]. Huang, J. and Li, K. (2008). A New Soy Flour-Based Adhesive for Making Interior Type II Plywood. Journal of the American Oil Chemists' Society, Vol. 85, No. 1.

[9]. Hussein, R. (2002). Exact Closed-Form Solutions for Fiber-Fiber and Bond-Fiber Interactions with Imperfect Bonding. Progress in Paper Physics Conference, Skaneateles NY.

[10].Hussein, R. (2002). An Analytic Solution for Fiber-Bond Interactions. The $116^{\text {th }}$ ESPRA Conference, Betlehem PA.

[11].Hussein, R. and Cheremisinoff, N. P. (1993). Polymer Applications in the Construction Industry. Chapter 26, Elasomer Technology Handbook, CRC Press.

[12].Hussein, R. (1993). Polymers in Sandwich Construction. Chapter 27, Elasomer Technology Handbook, CRC Press.

[13].Hussein, R.(1992). Effects of Bonding Stiffness on Thermal Stresses in Sandwich Panels. Journal of the Aerospace Engineering Division, ASCE, Vol. 5, No. 4.

[14].Hussein, R. et al (1989). Thermal Stresses in Sandwich Panels With Interlayer Slips. Journal of Thermal Stresses, Vol. 12 , No. 2.

[15].Hussein, R. (1986). Composite Panels/Plates - Analysis and Design. Technomic Publishing Inc. 
[16].Hussein, R. (1984). Sandwich Plates With Interlayer slips. Journal of the Engineering Mechanics Division, ASCE, Vol. 110, No. EM4.

[17].Hussein, R. (1984). Thermal Stresses in Sandwich Panels. Canadian Aeronautics and Space Journal, Vol. 30, No. 2.

[18].Hussein, R. et al (1982). Sandwich Beam-Columns With Interlayer Slips. Journal of the Engineering Mechanics Division, ASCE, Vol. 108, No. EM2.

[19].Hussein, R. (1980). Structural Behavior of Sandwich Panels. Doctoral Thesis, Concordia University, Canada.

[20].Hussein, R. (1978). Thermal Stresses in Flat Metal Face Sandwich Panel. Master Thesis, Concordia University, Canada.

[21].Isakson, G. and Levy, A. (1971). Finite Element Analysis of Interlaminar Shear in Fibrous Composites. Journal of Composite Materials, Vol. 5.

[22].Karbhari, V. M. (1997). Application of Composite Materials to the Renewal of Twenty-First Century Infrastructure. Proceedings of the Eleventh Conference on Composite Materials," Melbourne Australia.

[23].Kepler, J.A. and Hansen, M. R. (2007). Numerical Modeling of Sandwich Panel Response to Ballistic Loading - Energy Balance for Varying Impactor Geometries. No. 9.

[24].Mohanty, A. et. al. (2005). Natural Fibers, Biopolymers, and Biocomposites. CRC Press, Florida.

[25].Noor, A. K. et al. (1996). Computational Models for Sandwich Panels and Shells. Applied Mechanics Reviews, Vol. 49 , No. 3.

[26].Plantema, F. J. (1966). Sandwich Construction - The Bending and Buckling of Sandwich Beams, Plates and Shells. John Wiley and Sons, Inc.

[27].Puppo, A. H., and Evensen, H. A. (1970). Interlaminar Shear in Laminated Composites under Generalized Plane Stress,” Journal of Composite Materials, Vol. 4.

[28].Rigoberto, B. et. al. (2005). Sustainable Cellular Biocomposites from Natural Fibers and Unsaturated Polyester Resin for Housing Panel Applications. Journal of Polymers and the Environment, Vol. 13, No. 2.

[29].Satyanarayana, K. G. (2007). Biodegradable Composites based onlignocellulosicFibers. International Conference on Advanced Materials and Composites (ICAMC).

[30].Swanson, S. R. (1997). Introduction to Design and Analysis with advanced Composite Materials, Prentice-Hall Publisher.

[31].The University of Maine (2010), "Advanced Structures and Composite Center,"http://www.aewc.umaine.edu/.

[32].Vanderbilt, M. D., Goodman, J. R., and Criswell, M. E. (1974). Service and Overload Behavior of Wood Joist Floor Systems. Journal of the Structural Engineering Division, ASCE, Vol. 100, No. ST1.

[33].Zhu, F., et. al. (2009). A numerical simulation of the blast impact of square metallic sandwich panels," International Journal of Impact Engineering, Vol. 36.

\section{NOTATION}

$\begin{array}{ll}\mathrm{A}_{\mathrm{mn}} & =\text { Fourier coefficient; } \\ 2 \mathrm{a} & =\text { plate length in the x direction; } \\ 2 \mathrm{~b} & =\text { plate width in the y direction; } \\ \mathrm{C}_{\mathrm{mn}} & =\text { Fourier coefficient; } \\ \mathrm{c} & =\text { Subscript denoting flute; } \\ \mathrm{E} & =\text { Young's Modulus; } \\ \mathrm{f} & =\text { Subscript denoting face; } \\ \mathrm{G} & =\text { Shear Modulus; } \\ \mathrm{i} & =\text { Subscript denoting } \mathrm{x} \text { or } \mathrm{y} ; \\ \mathrm{K}_{\mathrm{i}} & =\text { Stiffness of bonding in the i direction; } \\ \mathrm{m}, \mathrm{n} & =\text { Summation counter; } \\ \mathrm{o} & =\text { Subscript denoting edge quantity; } \\ \mathrm{P} & =\text { Load density; } \\ \mathrm{q} & =\text { Interlayer shear stress; } \\ \mathrm{t}_{\mathrm{c}} & =\text { The thickness of the flute; } \\ \mathrm{t}_{\mathrm{f}} & =\text { The thickness of the face; } \\ \mathrm{u} & =\text { Displacement in the } \mathrm{x} \text { direction; } \\ \mathrm{v} & =\text { Displacement in the y direction; } \\ \mathrm{x}, \mathrm{y} & =\text { Coordinate axes. } \\ \Delta_{\mathrm{i}} & =\text { Interlayer deformation in the i direction; } \\ \varepsilon & =\text { Normalstrai; } \\ \varepsilon \text { and } \gamma & =\text { Shear strain; } \\ \mathrm{v} & =\text { Poisson's ratio; } \\ \sigma & =\text { Normal stress; } \\ \tau & =\text { Shear stress. }\end{array}$

